\title{
Expression of delta-catenin is associated with progression of human astrocytoma
}

Wang MingHao', Dong Qianze ${ }^{2}$, Zhang $\mathrm{Di}^{2}$ and Wang YunJie ${ }^{1 *}$

\begin{abstract}
Background: $\delta$-Catenin (CTNND2), which encodes a scaffold protein in humans, has been found in a few malignancies. However, the expression pattern and contribution of $\delta$-catenin to astrocytoma progression are unclear.
\end{abstract}

Methods: We investigated $\delta$-catenin expression in human astrocytoma samples and its function in astrocytoma cell lines using immunohistochemistry, siRNA knockdown, transfection, MTT, transwell migration and Rac1 pulldown techniques.

Results: $\delta$-Catenin protein expression was detected in cytoplasm of astrocytoma cells by immunohistochemistry. Analysis showed that grade I astrocytoma $(0 \%, 0 / 11)$ and glial cells from normal brain tissue exhibited negative staining. $\delta$-Catenin expression was significantly higher in grade III-IV $(35 \%, 29 / 84)$ compared to grade II astrocytoma cells $(18 \%, 11 / 61) ; p<0.01)$. In addition, CTNND2 overexpression promoted proliferation, invasion and Rac1 activity of U251 astrocytoma cells. Treatment of $\delta$-catenin-transfected cells with a Rac1 inhibitor decreased Rac1 activity and invasion. $\delta$-Catenin knockdown in U87 glioblastoma cell decreased cell proliferation, invasion and Rac1 activity.

Conclusion: The results suggest that $\delta$-catenin expression is associated with the malignant progression of astrocytoma and promotes astrocytoma cell invasion through upregulation of Rac 1 activity. $\delta$-Catenin expression levels may serve as a useful marker of the biological behavior of astrocytoma cells.

\section{Background}

Astrocytoma arises from neural stem or progenitor cells in the central nervous system. It is the most common primary brain tumor and accounts for approximately $60 \%$ of all brain tumors. Despite combined treatment strategies, which include surgery, radiotherapy and chemotherapy, the prognosis for high-grade astrocytoma, especially glioblastoma multiforme, has changed little over the past 10 years, with a median survival of only approximately 1 year [1]. The clinical symptoms and prognosis are closely correlated with tumor location and size and histological grade. Although the histological grade partly reflects the malignant features of astrocytoma, it cannot give an indication of the exact mechanism of tumor invasion and recurrence $[2,3]$. Thus, it is important to understand the molecular mechanism of astrocytoma cell invasion and identify effective markers in tumorigenesis and progression.

\footnotetext{
* Correspondence: wangyunjiepaper@yahoo.com.cn

${ }^{1}$ Department of Neurosurgery, First Affiliated Hospital of China Medical University, Shenyang 110001, PR China Full list of author information is available at the end of the article
}

The expression of $\delta$-catenin was initially found to be limited to brain neurons and it can bind to presenilin 1 , which is involved in the progression of Alzheimer's disease [4-6]. $\delta$-Catenin was also implicated in the maintenance of synaptic function and transmission of downstream signals as a scaffold protein [7-11]. It was reported that $\delta$-catenin overexpression changed the morphology of MDCK cells, including the elaboration of lamellipodia [12]. Furthermore, $\delta$-catenin can regulate the activity of small GTPases and therefore induces dendritic protrusions from cells [13]. Small GTPases are critical mediators of cytoskeletal reorganization, signal transduction and gene expression pathways [14,15]. As one of these GTPases, Rac1 is a pleiotropic regulator of many cellular processes, including the cell cycle, cell-cell adhesion, motility (through the actin network) and epithelial differentiation. $\delta$-Catenin also affects invasion and metastasis of lung cancer cells via regulation of small GTPase activity [16]. These studies suggest that $\delta$-catenin plays an important role in many human cancers. Our previous study showed that $\delta$-catenin was overexpressed in non-small-cell lung cancer and
Ciomed Central 
enhanced cancer cell invasion through small GTPase regulation. However, the expression pattern of $\delta$-catenin in glial cells and human astrocytoma cells is not clear; the relationship between its expression and clinicopathological factors and biological behavior still needs to be addressed.

In the present study, we examined the expression of $\delta$-catenin in 156 astrocytoma tissue specimens and analyzed the correlation between its expression and clinicopathological factors. We also investigated the effect of $\delta$-catenin on Rac1 activity and the biological behavior of astrocytoma cell lines.

\section{Methods}

\section{Patients and specimens}

The study protocol was approved by the institutional review board of China Medical University. Primary tumor specimens and adjacent normal brain tissue were obtained from 156 patients diagnosed with astrocytoma who underwent resection in the First Affiliated Hospital of China Medical University between 2005 and 2008. None of the patients had received radiotherapy or chemotherapy before surgical resection. There were 95 male (60.9\%) and 61 female (39.1\%) patients with a median age of 43 years (range 15-67 years). The histological diagnosis and differentiation grade were evaluated for sections stained with hematoxylin and eosin according to the World Health Organization (WHO) classification guidelines. All 156 specimens were re-evaluated with respect to histological subtype and tumor grade. Tumors were graded and classified into grade I (11), grade II (61), grade III (51), and grade IV (33) according to WHO guidelines (2007).

\section{Cell culture and transfection}

U251 and U87 cell lines were obtained from American Type Culture Collection (Manassas, VA, USA). The cells were cultured in DMEM (Invitrogen, Carlsbad, CA, USA) containing 10\% fetal calf serum (Invitrogen), $100 \mathrm{IU} / \mathrm{ml}$ penicillin (Sigma, St. Louis, MO, USA), and $100 \mu \mathrm{g} / \mathrm{ml}$ streptomycin (Sigma). Cells were grown on sterilized culture dishes and were passaged every 2 days with $0.25 \%$ trypsin (Invitrogen). The pCMV5-FLAG/ $\delta$-catenin plasmid was a kind gift from Dr. Shun-ichi Nakamura at Kobe University and was transfected into cells using Attractene Transfection (Qiagen, Hamburg, Germany). A pCMV5 empty vector was used as a negative control. DharmaFECT1 reagent was used for siRNA transfection (Qiagen, Chicago, IL, USA) according to the manufacturer's instructions. The protein level was assessed $48 \mathrm{~h}$ later by western blotting. The $\delta$-catenin siRNA sequences were 5'-CUA CGU UGA CUU CUA CUC AUU-3' and 5'-UGA GUA GAA GUC AAC GUA GUU-3' and were transfected into U87 cell lines, which express relatively high levels of $\delta$-catenin. Nonsilencing siRNA sequences were used as a negative control (5'-UUC UCC GAA CUU GUC ACA UUU-3' and 5'-AUG UGA CAA GUU CGG AGA AUU-3').

\section{Immunohistochemistry}

Surgically excised tumor specimens were fixed with $10 \%$ neutral formalin and embedded in paraffin, and 4- $\mu \mathrm{m}$ thick sections were prepared. Normal brain neurons present in the tumor slides was used as positive control. Immunostaining was performed using the avidin-biotinperoxidase complex method (Ultrasensitive ${ }^{\mathrm{TM}}$, MaiXin, Fuzhou, China). The sections were deparaffinized in xylene, rehydrated with graded alcohol, and then boiled in $0.01 \mathrm{M}$ citrate buffer ( $\mathrm{pH}$ 6.0) for $2 \mathrm{~min}$ in an autoclave. Hydrogen peroxide $(0.3 \%)$ was applied to block endogenous peroxide activity and the sections were incubated with normal goat serum to reduce nonspecific binding. Tissue sections were incubated with $\delta$-catenin mouse monoclonal antibody (1:100 dilution; Santa Cruz Biotechnology, Santa Cruz, CA, USA). Mouse immunoglobulin (at the same concentration as for the antigen-specific antibody) was used as a negative control. Staining for both antibodies was performed at room temperature for $2 \mathrm{~h}$. Biotinylated goat antimouse serum IgG was used as a secondary antibody. After washing, the sections were incubated with streptavidin-biotin conjugated with horseradish peroxidase, and the peroxidase reaction was developed with 3,3'diaminobenzidine tetrahydrochloride. Counterstaining with hematoxylin was performed and the sections were dehydrated in ethanol before mounting.

Two independent blinded investigators examined all tumor slides randomly. Five views were examined per slide, and 100 cells were observed per view at $400 \times$ magnification. Immunostaining of $\delta$-catenin was scored on a semiquantitative scale by evaluating in representative tumor areas the intensity and percentage of cells showing significantly higher immunostaining than control cells in normal brain tissues. Cytoplasmic immunostaining in tumor cells was considered positive staining. We counted 400 tumor cells and calculated the percentage of positively stained cells. The proportion of cells exhibiting $\delta$-catenin expression was categorized as follows: 0 , absent; $1,1-25 \%$; 2, 26-50\%; 3, 51-75\%; and 4, $\geq 76 \%$. The staining intensity was categorized as follows: 0 , negative; 1 , moderate; and 2, strong. The proportion and intensity scores were then multiplied to obtain a total score. To obtain final statistical results, a score $<2$ was considered negative and scores $\geq 2$ were considered positive.

\section{Western blot analysis}

Total proteins from tissue and cells were extracted in lysis buffer (Pierce, Rockford, IL) and quantified using the Bradford method. Samples of $50 \mu \mathrm{g}$ of protein were separated by SDS-PAGE. Samples were transferred to 
polyvinylidene fluoride membranes (Millipore, Billerica, MA, USA) and incubated overnight at $4^{\circ} \mathrm{C}$ with antibody against $\delta$-catenin (1:1000; Santa Cruz) and a mouse monoclonal antibody against $\beta$-actin (1:500; Santa Cruz). After incubation with peroxidase-coupled antimouse IgG (Santa Cruz) at $37^{\circ} \mathrm{C}$ for $2 \mathrm{~h}$, bound proteins were visualized using ECL (Pierce) and detected using a BioImaging System (UVP Inc., Upland, CA, USA). Relative protein levels were quantified using $\beta$ actin as a loading control.

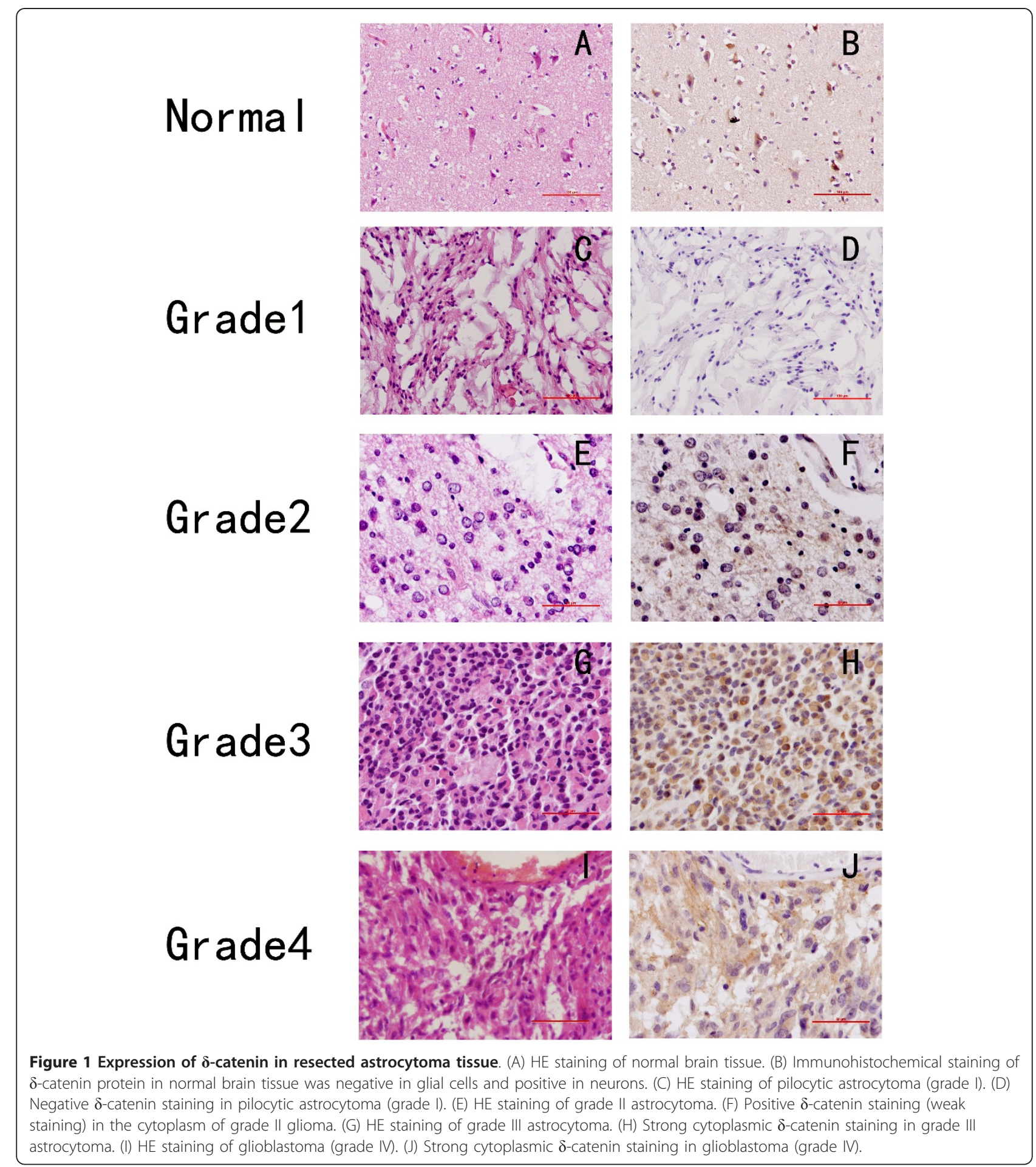




\section{Colony formation and MTT assays}

U251 and U87 cells were transfected with siRNA or a $\delta$ catenin plasmid for $48 \mathrm{~h}$ and then plated into three 6$\mathrm{cm}$ cell culture dishes (1000 per dish) and incubated for 12 days. Plates were washed with PBS and stained with Giemsa. The number of colonies with more than 50 cells was counted. The colonies were manually counted using a microscope.

Cells were plated in 96-well plates in medium containing $10 \% \mathrm{FBS}$ at approximately 3000 cells per well $24 \mathrm{~h}$ after transfection. For quantitation of cell viability, cultures were stained after 4 days in MTT assays. In brief, $20 \mu \mathrm{l}$ of $5 \mathrm{mg} / \mathrm{ml} \mathrm{MTT} \mathrm{(thiazolyl} \mathrm{blue)} \mathrm{solution} \mathrm{was}$ added to each well and incubated for $4 \mathrm{~h}$ at $37^{\circ} \mathrm{C}$. The medium was removed from each well and the resulting MTT formazan was solubilized in $150 \mu \mathrm{l}$ of DMSO. Each solution was measured spectrophotometrically at $490 \mathrm{~nm}$.

\section{Matrigel invasion assay}

Cell invasion assays were performed in 24-well Transwell chambers with a pore size of $8 \mu \mathrm{m}$ and the inserts were coated with $20 \mu \mathrm{l}$ of Matrigel (1:3 dilution, BD Bioscience, San Diego, CA, USA). At $48 \mathrm{~h}$ after transfection, cells were trypsinized and transferred to the upper Matrigel chamber in $100 \mu \mathrm{l}$ of serum-free medium containing $3 \times 10^{5}$ cells and incubated for $16 \mathrm{~h}$. Medium supplemented with $10 \%$ FBS was added to the lower chamber as the chemoattractant. Then non-invading cells on the upper membrane surface were removed with a cotton tip and cells that had passed through the filter were fixed with $4 \%$ paraformaldehyde and stained with hematoxylin. The number of invaded cells were counted in 10 randomly selected high-power fields under a microscope. The experiments were performed in triplicate.

\section{Rac1 pulldown assaym}

Rac1 activity was measured using a Active Rac1 PullDown and Detection Kit (Pierce). The Rac1 inhibitor NSC23766 was from Calbiochem (Merck, Whitehouse Station, NJ, USA). Cells were treated for $6 \mathrm{~h}$ at a concentration of $50 \mu \mathrm{M}$.

\section{Statistical analysis}

SPSS version 11.5 for Windows was used for all statistical analyses. A $\chi^{2}$ test was used to examine possible correlations between $\delta$-catenin expression and clinicopathologic factors. Student's t-test was used to compare densitometry data on focus numbers between control and $\delta$-catenin-transfected cells. All $\mathrm{p}$ values are based on a two-sided statistical analysis, and $p<0.05$ was considered to indicate statistical significance.
Table 1 The relationship between $\delta$-catenin and clinicopathological features

\begin{tabular}{|c|c|c|c|c|}
\hline \multirow[t]{2}{*}{ Clinical parameters } & \multirow[t]{2}{*}{ Number } & \multicolumn{2}{|l|}{$\boldsymbol{\delta}$-catenin } & \multirow[t]{2}{*}{$p$ value } \\
\hline & & Negative & Positive & \\
\hline \multicolumn{5}{|l|}{ Age } \\
\hline$<43$ & 78 & 61 & 17 & 0.359 \\
\hline$\geq 43$ & 78 & 55 & 23 & \\
\hline \multicolumn{5}{|l|}{ Gender } \\
\hline Male & 95 & 69 & 26 & 0.578 \\
\hline Female & 61 & 47 & 14 & \\
\hline \multicolumn{5}{|l|}{ Grading } \\
\hline Grade I & 11 & 11 & 0 & $0.009^{*}$ \\
\hline Grade II & 61 & 51 & 11 & \\
\hline High Grade (III-IV) & 84 & 55 & 29 & \\
\hline
\end{tabular}

\section{Results}

Expression and localization of $\delta$-catenin in normal brain tissue and astrocytoma cells

In cells from normal brain tissue, only neurons showed strong cytoplasmic (include axon) expression of $\delta$-catenin, whereas glial cells showed no expression (Figure 1a, b). Cytoplasmic staining was present in a subset of astrocytoma cases, while no staining was detected in sections from the same samples subjected to immunohistochemical analysis using mouse immunoglobulin. Of the 156 patients in the study, $40(25.6 \%)$ showed positive $\delta$-catenin expression. $\delta$-Catenin expression predominantly occurs in the cytoplasmic compartment of tumor cells. We analyzed the correlation between $\delta$-catenin protein expression and the histological staging of astrocytoma. As shown in Table 1 Grade I astrocytoma $(0 \%, 0 / 11)$ and normal glial cells exhibited negative staining (Figure 1c, d). In grade III-IV astrocytoma (35\%, 29/84; Figure 1g-j), $\delta$-catenin expression was significantly higher than in grade II astrocytoma

Table 2 Association of $\delta$-catenin expression with tumor location

\begin{tabular}{llll}
\hline & \multicolumn{3}{l}{$\boldsymbol{\delta}$-catenin } \\
\hline Sites & Total & Negative & Positive \\
\hline Supratentorial & & & \\
\hline Hemisphere & 124 & $87(70.2 \%)$ & $37(29.8 \%)$ \\
\hline Diencephalon & 2 & $2(100 \%)$ & $0(0 \%)$ \\
\hline Total & 126 & $89(70.7)$ & $37(29.3 \%)$ \\
\hline Infratentorial & & & \\
\hline Cerebellum & 17 & $13(76.5 \%)$ & $4(23.5 \%)$ \\
\hline Brain stem & 5 & $5(100 \%)$ & $0(0 \%)$ \\
\hline Spinal cord & 8 & $8(100 \%)$ & $0(0 \%)$ \\
\hline Total & 30 & $26(86.7 \%)$ & $4(13.3 \%)^{*}$ \\
\hline
\end{tabular}

* Supratentorial versus Infratentorial, $p<0.05$ 


\section{Delta catenin}

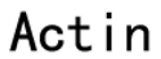

\section{KD}

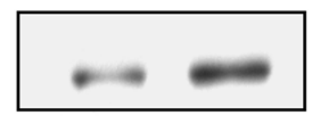

45KD

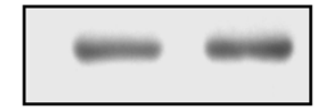

U251 U251

EV Delta
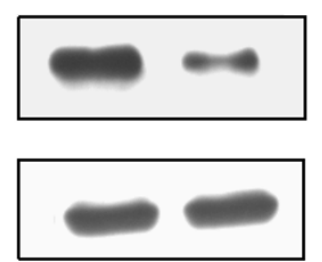

U87 U87

Neg Delta

siRNA catenin

s iRNA
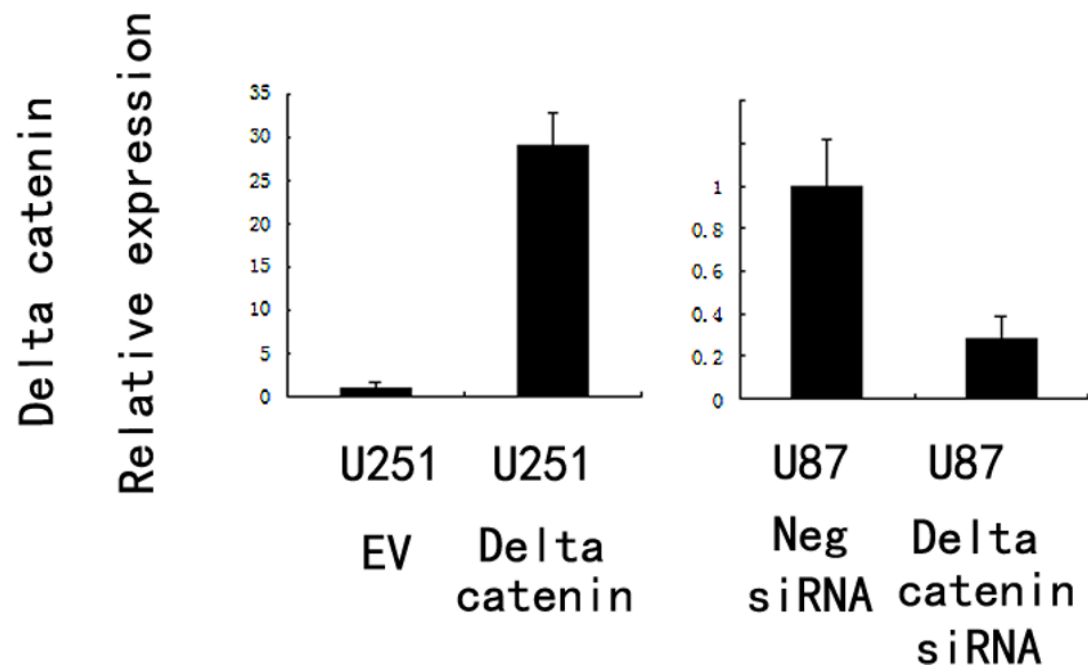

Figure $2 \boldsymbol{\delta}$-Catenin transfection of $\mathbf{0} \mathbf{2 5 1}$ cells and siRNA knockdown in U87 cells. Western blot and real-time PCR analyses showed that U251 cells transfected with the $\delta$-catenin plasmid expressed higher levels of $\delta$-catenin. $\delta$-Catenin expression in U87 cells transfected with siRNA was downregulated.

$(18 \%, 11 / 61$; Figure 1e, f, p $<0.01)$, which suggests that $\delta$ catenin expression is associated with astrocytoma progression.

We examined the difference between the expression pattern of $\delta$-catenin according to tumor location. As shown in Table 2 the positive rate of $\delta$-cateninin supratentorial astrocytomas was $29.3 \%$, much higher than the rate in infratentorial astrocytomas (13.3\%). $(p<0.05)$.

\section{$\boldsymbol{\delta}$-Catenin overexpression promotes astrocytoma cell proliferation and invasion via Rac1}

To determine whether $\delta$-catenin enhances the proliferation and invasion of astrocytoma cells, we carried out $\delta$ catenin transfection in the U251 astrocytoma cell line. siRNA knockdown was performed in the U87 glioblastoma cell line.

As shown in Figure 2, $\delta$-catenin transfection considerably increased protein levels at $48 \mathrm{~h}$ after treatment, whereas $\delta$-catenin siRNA knockdown decreased $\delta$-catenin protein in U87 cells. The proliferation rate was determined by MTT assay. A significant increase in proliferation was observed in U251 cells overexpressing $\delta$-catenin compared to cells transfected with the empty vector (Figure 3a). Consistent with the MTT results, colony formation assays showed that transfection of $\delta$-catenin in U251 cells led to an increase in focus numbers (empty vector $109 \pm 23$ vs $\delta$ catenin $174 \pm 26, p<0.05$; Figure $3 b)$. $\delta$-Catenin also promoted tumor cell invasion in a Matrigel assay (empty vector $55 \pm 10$ vs $\delta$-catenin $73 \pm 15, p<0.05$; Figure $4 \mathrm{a}$ ). In U251 cells overexpressing $\delta$-catenin, immunoblotting showed total Rac1 protein levels were unchanged, but a pulldown assay revealed that Rac1 activity increased significantly (Figure 4b). Conversely, $\delta$-catenin knockdown decreased the proliferation, colony formation ability (control158 \pm 21 vs siRNA $\delta$-catenin $97 \pm 17, p<0.05$; Figure 5 ) and invasiveness of U87 cells (control $59 \pm 7$ vs siRNA $\delta$-catenin $36 \pm 5, p<0.05)$. $\delta$-Catenin knockdown also decreased Rac1 activity (Figure 6). 


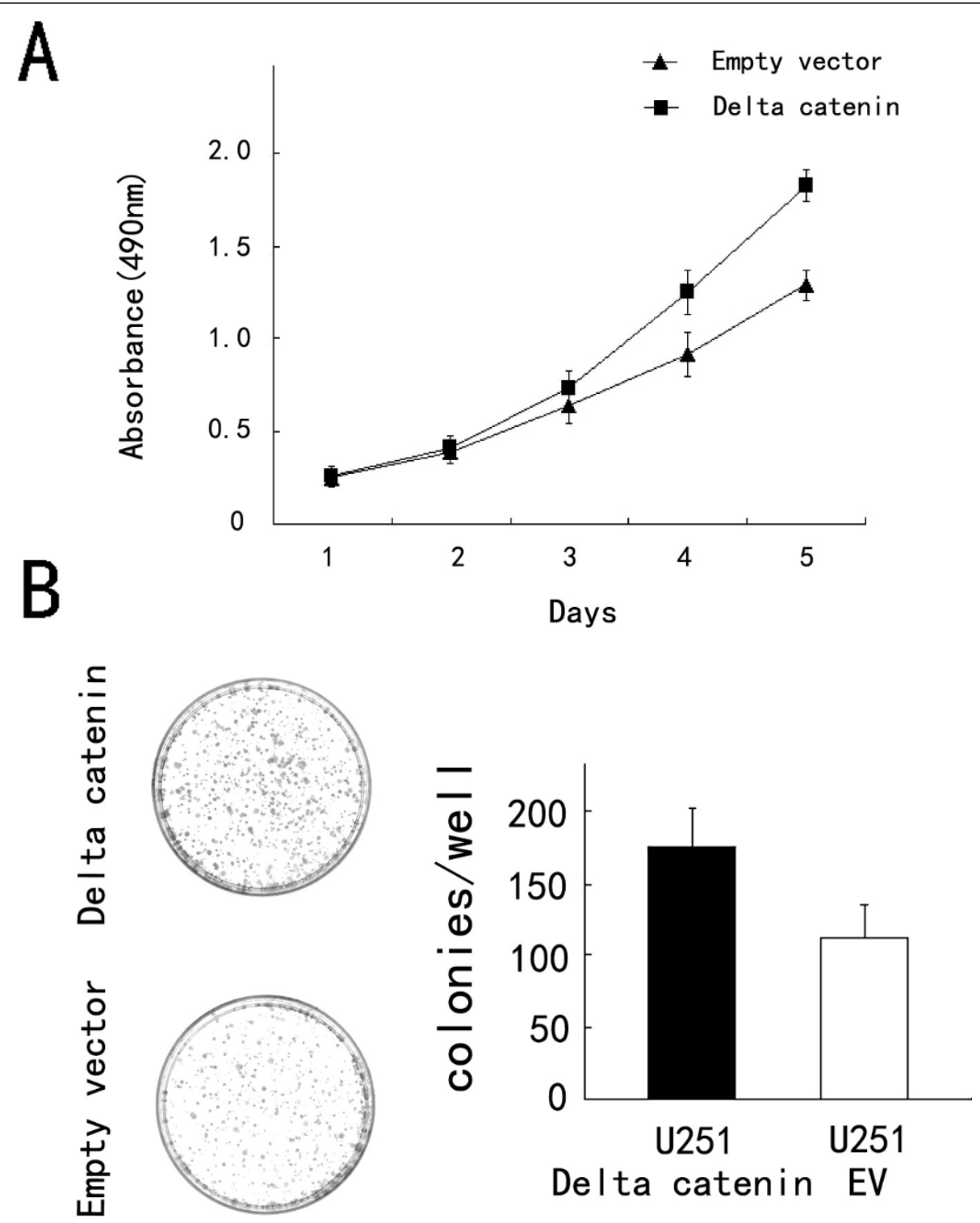

Figure $3 \boldsymbol{\delta}$-Catenin transfection promotes cancer cell proliferation in U251 cells. (A) MTT assays were performed after $\delta$-catenin transfection. An increase in absorbance was observed. (B) Assessment of the clonogenic potential of $\delta$-catenin-transfected glioma cells. The number of colonies formed by cells treated with $\delta$-catenin was greater than for control cells $(p<0.05)$. Columns, mean; bars, SD.

To explore whether the effect of $\delta$-catenin on cell invasion was dependent on Rac1 activity, we measured cell invasion and Rac1 activity after treatment of $\delta$-catenintransfected cells with the Rac1 inhibitor NSC23766. The inhibitor abolished the effect of $\delta$-catenin on cell invasion $(\delta$-catenin $73 \pm 15$ vs $\delta$-catenin + Rac1 inhibitor $42 \pm 9$, $p<0.05)$.

\section{Discussion}

$\delta$-catenin plays an important role in dendritic morphogenesis, which is associated with changes in the activity of small GTPases $[7,13]$. The formation and number of dendritic protrusions are indicative of the malignant phenotype of cells, so it is possible that $\delta$-catenin promotes a malignant phenotype in tumor cells. It has been reported that $\delta$-catenin expression was increased in over $80 \%(55 / 65)$ of prostatic adenocarcinomas, and was associated with higher Gleason scores [4]. Some researchers also observed gene amplification of $\delta$-catenin in cervical and bladder carcinoma. $\delta$-catenin mRNA is probably present in esophagus, ovary and breast tumors $[17,18]$. Our previous study showed that $\delta$-catenin was overexpressed in non-small-cell lung cancer and was correlated with clinicopathological factors [16]. However, the expression pattern and clinical significance of $\delta$-catenin are poorly defined in other human cancers such as astrocytoma. 

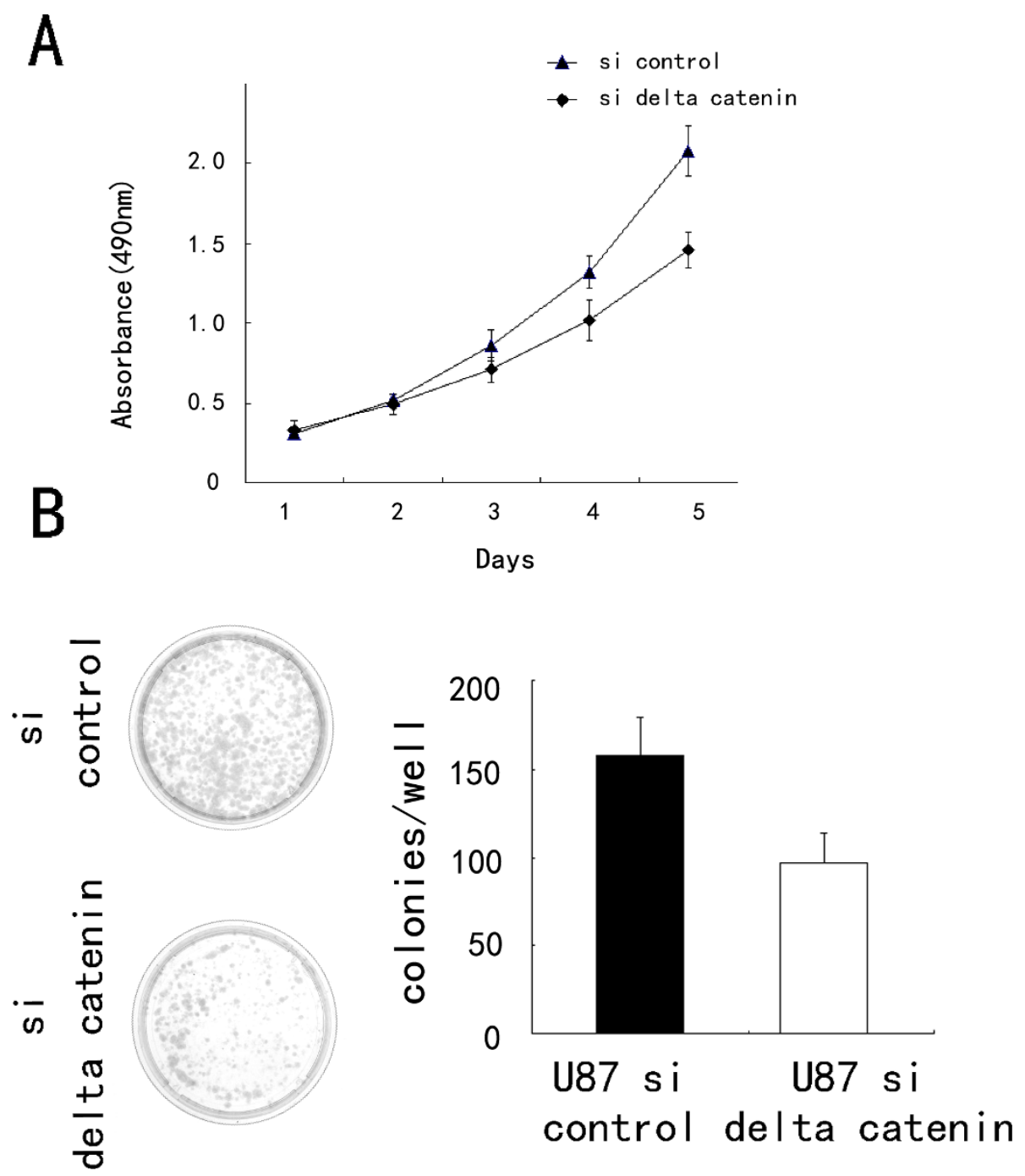

Figure $4 \boldsymbol{\delta}$-Catenin knockdown inhibits proliferation in U87 cells. (A) MTT assays were performed after $\delta$-catenin siRNA transfection. A decrease in absorbance was observed. (B) Assessment of the clonogenic potential of $\delta$-catenin-transfected glioma cells. The number of colonies formed by cells treated with $\delta$-catenin siRNA was less than for control cells $(p<0.05)$. Columns, mean; bars, SD.

Analysis of mRNA in primary cultures of neurons and glial cells prepared from fetal rat brain showed that $\delta$ catenin expression levels were higher in neurons than in glial cells [19]. However, its protein expression in neurons and glial cells has not been investigated. In this study, we found strong cytoplasmic $\delta$-catenin expression in neurons but negative staining in human glial cells such as astrocytes, which may due to the low mRNA copy numbers of $\delta$-catenin. Although there was no expression of $\delta$-catenin in 11 grade I astrocytoma cases, positive $\delta$ catenin was found in $18 \%$ of grade II and $35 \%$ of grade III-IV astrocytoma cases, which suggests that $\delta$-catenin is positively associated with higher grade astrocytoma. Positive rate of $\delta$-catenin in low grade astrocytoma is very low, especially in grade I astrocytoma (Negative expression). and its utilization in the distinguishing grade I astrocytoma from reactive gliosis was limited. The low grade astrocytoma with positive $\delta$-catenin expression may transform into high grade astrocytoma. These findings indicate that $\delta$-catenin might play an important role in astrocytoma progression and could serve as a potential biomarker for the biological behavior of astrocytoma cells.

To explore the relationship between $\delta$-catenin and the biological behavior of astrocytoma cells, we transfected a $\delta$ catenin plasmid into the U251 cell line, which has low $\delta$ catenin expression, and knocked down $\delta$-catenin in the U87MG cell line, which has high $\delta$-catenin expression. We found that $\delta$-catenin overexpression caused a significant increase in the proliferation rate, colony formation and 


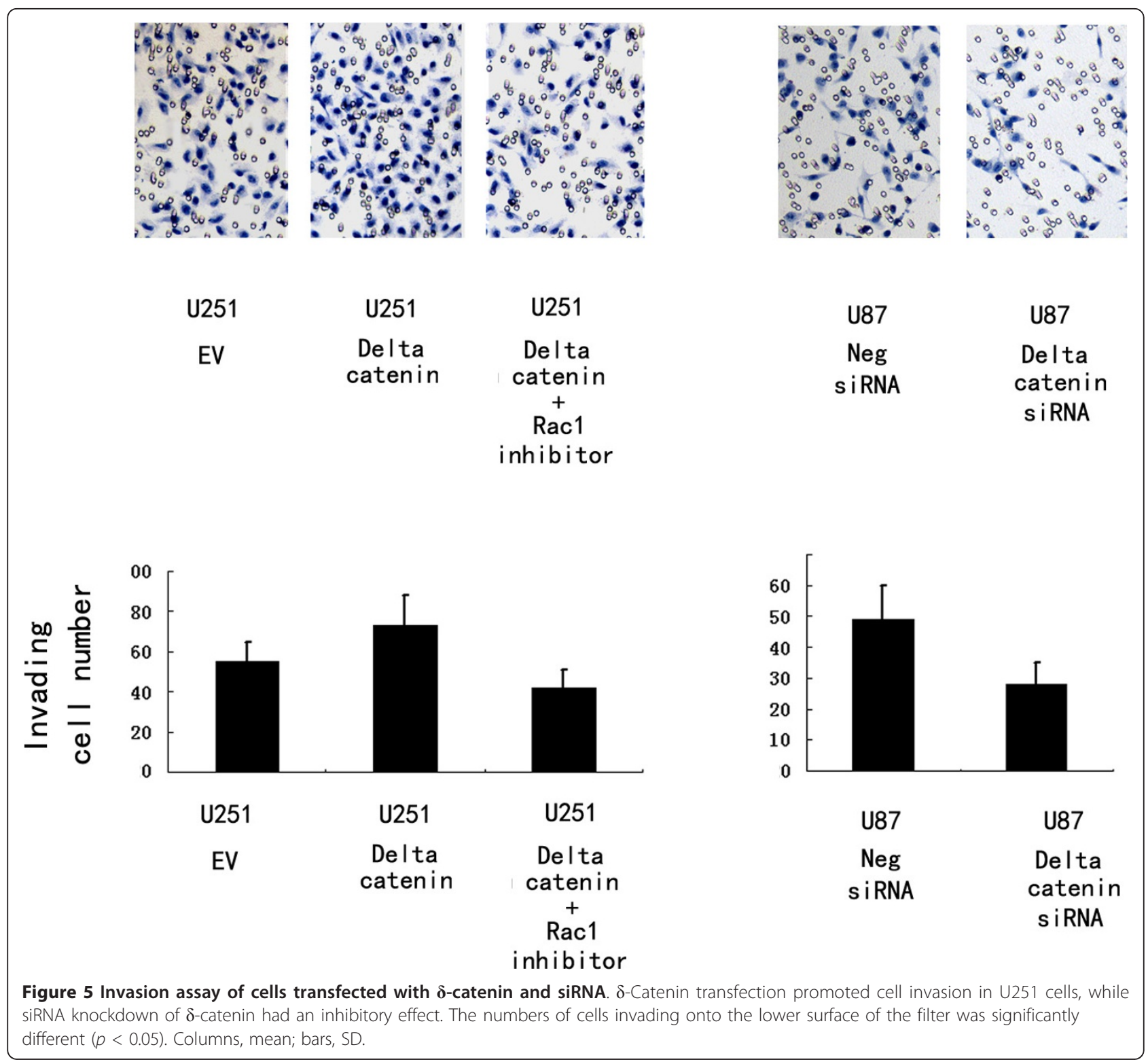

invasion ability of U251 cell lines, with a significant increase in Rac1 activity, which was in accordance with our previous results for lung cancer [16]. On the contrary, $\delta$-catenin knockdown decreased the proliferation rate, colony formation ability and invasiveness of U87 cells, with a decrease in Rac1 activity. We then observed a decrease in invasiveness and Rac 1 activity after treatment of $\delta$-catenin-transfected cells with a Rac1 inhibitor, which demonstrates that $\delta$-catenin regulates cell invasion in a Rac1-dependent manner. These results suggest that $\delta$-catenin might enhance the invasive ability of astrocytoma cells via Rac1. However, the exact mechanism of Rac1 regulation by $\delta$-catenin is not clear. In addition, previous studies proved that $\delta$-catenin has an influence in other small GTPases, such as Cdc42 and RhoA, which was not investigated in glioma cell lines [13]. Further studies are required to address these issues.

\section{Conclusion}

In conclusion, overexpression of $\delta$-catenin in astrocytoma cells was correlated with higher grade and $\delta$-catenin was identified as an important oncoprotein in maintaining the malignant behavior of glioma cells. Overexpression of $\delta$-catenin activated Rac 1 and promoted astrocytoma cell proliferation and invasion. Thus, $\delta$-catenin is a candidate target protein for future astrocytoma therapeutics. 


\section{Active Rac1 \\ Total Rac1}

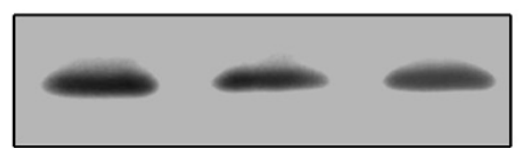

\section{U251 U251 U251 EV}
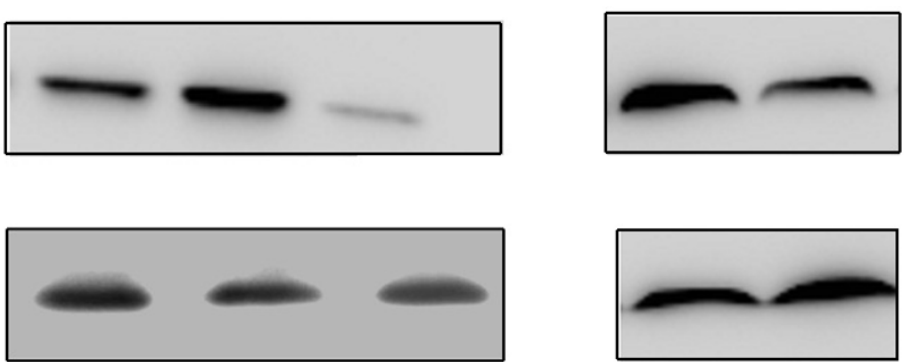

U87 U87

Neg Delta

siRNA catenin

s iRNA

Rac1

\section{inhibitor}

Figure $6 \boldsymbol{\delta}$-Catenin transfection and siRNA knockdown affect Rac1 activity. Overexpression of the $\delta$-catenin gene promoted Rac1 activity in U251 astrocytoma cells. siRNA treatment and a Rac1 inhibitor decreased this activity.

\section{Acknowledgements}

We thank Dr. Shun-ichi Nakamura at Kobe University, Japan, for kindly providing $\mathrm{pCMV5-FLAG/ \delta}$-catenin plasmid.

\section{Author details}

'Department of Neurosurgery, First Affiliated Hospital of China Medical University, Shenyang 110001, PR China. ${ }^{2}$ Department of Pathology, First Affiliated Hospital of China Medical University, Shenyang, PR China.

\section{Authors' contributions}

WM and ZD carried out the immunohistochemistry, cell biological analysis and immunoassay. DQ participated in the design of the study and drafted the manuscript. WY participated in its design. All authors read and approved the final manuscript.

\section{Competing interests}

The authors declare that they have no competing interests.

Received: 19 June 2011 Accepted: 12 December 2011 Published: 12 December 2011

\section{References}

1. Bondy ML, Scheurer ME, Malmer B, Barnholtz-Sloan JS, Davis FG, Il'yasova D, Kruchko C, McCarthy BJ, Rajaraman P, Schwartzbaum JA, Sadetzki S, Schlehofer B, Tihan T, Wiemels JL, Wrensch M, Buffler PA: Brain tumor epidemiology: consensus from the Brain Tumor Epidemiology Consortium. Cancer 2008, 113(7 Suppl):1953-1968.

2. Walker PR, Calzascia T, de Tribolet N, Dietrich PY: T-cell immune responses in the brain and their relevance for cerebral malignancies. Brain Res Brain Res Rev 2003, 42(2):97-122.

3. van den Bent MJ, Hegi ME, Stupp R: Recent developments in the use of chemotherapy in brain tumours. Eur J Cancer 2006, 42(5):82-588.

4. Lu Q, Dobbs LJ, Gregory CW, Lanford GW, Revelo MP, Shappell S, Chen YH: Increased expression of delta-catenin/neural plakophilin-related armadillo protein is associated with the down-regulation and redistribution of E-cadherin and p120ctn in human prostate cancer. Hum Pathol 2005, 36(10):1037-1048.

5. Tanahashi H, Tabira T: Isolation of human delta-catenin and its binding specificity with presenilin 1. Neuroreport 1999, 10(3):563-568.
6. Zhou J, Liyanage U, Medina M, Ho C, Simmons AD, Lovett M, Kosik KS: Presenilin 1 interaction in the brain with a novel member of the Armadillo family. Neuroreport 1997, 8(8):2085-2090.

7. Kim H, Han JR, Park J, Oh M, James SE, Chang S, Lu Q, Lee KY, Ki H, Song WJ, Kim K: Delta-catenin-induced dendritic morphogenesis. An essential role of p190RhoGEF interaction through Akt1-mediated phosphorylation. J Biol Chem 2008, 283(2):977-987.

8. Lu Q, Mukhopadhyay NK, Griffin JD, Paredes M, Medina M, Kosik KS: Brain armadillo protein delta-catenin interacts with Abl tyrosine kinase and modulates cellular morphogenesis in response to growth factors. Neurosci Res 2002, 67(5):618-624.

9. Ochiishi T, Futai K, Okamoto K, Kameyama K, Kosik KS: Regulation of AMPA receptor trafficking by delta-catenin. Mol Cell Neurosci 2008, 39(4):499-507.

10. Arikkath J, Reichardt LF: Cadherins and catenins at synapses: roles in synaptogenesis and synaptic plasticity. Trends Neurosci 2008, 31(9):487-494

11. Kim K, Sirota A, Chen Yh YH, Jones SB, Dudek R, Lanford GW, Thakore C, Lu Q: Dendrite-like process formation and cytoskeletal remodeling regulated by delta-catenin expression. Exp Cell Res 2002, 275(2):171-184.

12. Lu Q, Paredes M, Medina M, Zhou J, Cavallo R, Peifer M, Orecchio L, Kosik KS: delta-catenin, an adhesive junction-associated protein which promotes cell scattering. J Cell Biol 1999, 144(3):519-532.

13. Abu-Elneel K, Ochiishi T, Medina M, Remedi M, Gastaldi L, Caceres A, Kosik KS: A delta-catenin signaling pathway leading to dendritic protrusions. J Biol Chem 2008, 283(47):32781-91.

14. Vega FM, Ridley AJ, SnapShot: Rho family GTPases. Cell 2007, 129(7):1430

15. Sahai E, Marshall CJ: RHO-GTPases and cancer. Nat Rev Cancer 2002, 2(2):133-142.

16. Zhang JY, Wang Y, Zhang D, Yang ZQ, Dong XJ, Jiang GY, Zhang PX, Dai SD, Dong QZ, Han Y, Zhang S, Cui QZ, Wang EH: delta-Catenin promotes malignant phenotype of non-small cell lung cancer by noncompetitive binding to E-cadherin with p120ctn in cytoplasm. J Pathol 222(1):76-88.

17. Zheng M, Simon R, Mirlacher M, Maurer R, Gasser T, Forster T, Diener PA, Mihatsch MJ, Sauter G, Schraml P: TRIO amplification and abundant mRNA expression is associated with invasive tumor growth and rapid tumor cell proliferation in urinary bladder cancer. Am J Pathol 2004, 165(1):63-69.

18. Huang FY, Chiu PM, Tam KF, Kwok YK, Lau ET, Tang MH, Ng TY, Liu WW, Cheung AN, Ngan HY: Semi-quantitative fluorescent PCR analysis 
identifies PRKAA1 on chromosome 5 as a potential candidate cancer gene of cervical cancer. Gynecol Oncol 2006, 103(1):219-225.

19. Kawamura Y, Fan QW, Hayashi H, Michikawa M, Yanagisawa K, Komano H: Expression of the mRNA for two isoforms of neural plakophilin-related arm-repeat protein/delta-catenin in rodent neurons and glial cells. Neurosci Lett 1999, 277(3):185-188.

\section{Pre-publication history}

The pre-publication history for this paper can be accessed here: http://www.biomedcentral.com/1471-2407/11/514/prepub

doi:10.1186/1471-2407-11-514

Cite this article as: MingHao et al:. Expression of delta-catenin is associated with progression of human astrocytoma. BMC Cancer 2011

11:514.

Submit your next manuscript to BioMed Central and take full advantage of:

- Convenient online submission

- Thorough peer review

- No space constraints or color figure charges

- Immediate publication on acceptance

- Inclusion in PubMed, CAS, Scopus and Google Scholar

- Research which is freely available for redistribution

Submit your manuscript at www.biomedcentral.com/submit 